\title{
Can drug-induced sleep endoscopy improve the success rates of tongue base surgery?
}

Jong-Gyun Ha'1, Youngwoo Lee', Jae Sung Nam¹, Jeong Jin Park', Joo-Heon Yoon 1,2,3, Chang-Hoon Kim,2,3,4 and Hyung-Ju Cho ${ }^{1,2,3^{*}}$

\begin{abstract}
Background: The purpose of this study was to determine the therapeutic value of drug-induced sleep endoscopy (DISE) by comparing the outcomes of tongue base surgery based on Muller's maneuver (MM) and those based on DISE in obstructive sleep apnea (OSA) patients.

Methods: Ninety-five patients who underwent the tongue base surgery in combination with palatal surgery for OSA at a tertiary referral hospital between March 2012 and March 2019 were enrolled in this retrospective comparative study. Forty-seven patients underwent MM for surgical decision and 48 patients underwent DISE in addition to MM for surgical decision. Surgical success was defined according to the Sher criteria (postoperative apnea-hypopnea index $[\mathrm{AHI}]<20 / \mathrm{h}$ and $\geq 50 \%$ reduction in preoperative $\mathrm{AHI}$ ), and $\mathrm{AHI}$ improvement (\%) was defined as (preoperative AHI-postoperative AHI) $\times 100$ /preoperative AHI. For comparison between the MM and DISE groups, $p$-values were calculated using independent or paired t-tests for continuous variables and using chisquare test for categorical variables.
\end{abstract}

Results: By comparing the results of MM and DISE, consensus on the tongue base level showed insignificant concordance $(\mathrm{kappa}=0.017, p=0.865)$, whereas that on the oropharynx level showed fair agreement (kappa $=$ $0.241, p=0.005)$. AHI, supine $A H I$, rapid eyeball movement (REM) AHI, non-REM AHI, and nadir oxygen saturation were all significantly improved after the tongue base surgery in both groups. The MM group showed a significant improvement in the Epworth sleepiness scale after the tongue base surgery $(p=0.014)$, whereas the DISE group did not $(p=0.165$ ). However, there was no significant difference in the AHI improvement (MM group $=47.0 \pm 32.0$, DISE group $=48.3 \pm 35.4, p=0.852)$ and surgical success (MM group $=42.6 \%$, DISE group $=45.8 \%, p=0.748)$ between the groups. Tonsil grade $(p<0.05)$ and occlusion at the oropharynx lateral wall $(p=0.031)$ were significantly related to surgical success in the MM group.

Conclusions: In the judgment of the tongue base surgery, MM and DISE findings showed poor agreement. DISE might affect the surgical decision on the tongue base surgery in OSA patients; however, there was a lack of evidence regarding the superiority of DISE over MM with respect to the surgical outcomes.

Keywords: Obstructive sleep apnea, Drug-induced sleep endoscopy, Muller maneuver, Tongue base surgery

\footnotetext{
* Correspondence: hyungjucho@yuhs.ac

'Department of Otorhinolaryngology, Yonsei University College of Medicine,

50-1 Yonsei-ro, Seodaemun-gu, Seoul 03722, South Korea

${ }^{2}$ The Airway Mucus Institute, Yonsei University College of Medicine, Seoul,

South Korea

Full list of author information is available at the end of the article
}

(c) The Author(s). 2020 Open Access This article is distributed under the terms of the Creative Commons Attribution 4.0 International License (http://creativecommons.org/licenses/by/4.0/), which permits unrestricted use, distribution, and reproduction in any medium, provided you give appropriate credit to the original author(s) and the source, provide a link to the Creative Commons license, and indicate if changes were made. The Creative Commons Public Domain Dedication waiver (http://creativecommons.org/publicdomain/zero/1.0/) applies to the data made available in this article, unless otherwise stated. 


\section{Background}

Obstructive sleep apnea (OSA) is a syndrome characterized by repetitive episodes of complete or partial collapse of the upper airway during sleep resulting in cessation or reduction of airflow and significant oxygen desaturation [1]. In general, the optimal treatment method for OSA is determined according to the patient's anatomical structures (tongue base, tonsil, soft palate, adenoid, nasal septum, and inferior turbinate), polysomnographic results, and personal preferences [2]. Continuous positive airway pressure (CPAP) therapy can reduce the risk of cardiovascular comorbidity in OSA patients [3] and is considered the first therapeutic option for OSA patients. However, other therapeutic options, including surgery, are considered for CPAP-intolerant OSA patients [4].

Pre-operative physical evaluation with nasopharyngoscopy is useful to determine the level or pattern of airway collapse. Traditional nasopharyngoscopic examination of the upper airway is generally performed during the awake state. Muller's maneuver (MM) is an established and noninvasive flexible endoscopic technique that can be performed easily at an outpatient clinic while the patient is awake [5]. However, the technique does not reflect the actual status of the upper airway during the sleep state of a patient. Since Croft and Pringle introduced drug-induced sleep endoscopy (DISE) in 1991, it has been used widely to evaluate the upper airway under similar conditions to sleeping status [6]. Several studies have attempted to compare the results of DISE with those of awake examination performed via MM. According to recent studies, the relation of obstruction of the lateral wall and that at the retropalatal level showed relatively high conformity between MM and DISE; however, obstruction at the tongue base level showed a lower consensus $[7,8]$. In some recent studies, about 40 $50 \%$ of the surgical plan based on awake examination changed after performing DISE, especially at the tongue base level $[9,10]$. Therefore, DISE may be more effective and efficient for evaluating obstruction at the tongue base level in OSA patients than conventional MM. However, it remains unclear whether surgical treatment based on DISE yields better results than that based on MM.

Therefore, the purpose of this study was to determine the therapeutic value of DISE by comparing the outcomes of tongue base surgery in OSA patients based on the findings of MM and DISE.

\section{Methods}

\section{Patient profiles}

From January 2013 to March 2019, we retrospectively reviewed the medical records of patients who underwent sleep surgery for OSA at Severance Hospital, a tertiary referral hospital in South Korea. Inclusion criteria for patients were as follows: (1) age $\geq 19$ years (2); diagnosed with OSA (apnea-hypopnea index $[\mathrm{AHI}] \geq 5 / \mathrm{h}$ ) using overnight polysomnography (PSG) (3); non-compliant or refused CPAP therapy, as a nonsurgical treatment (4); underwent tongue base surgery (e.g., tongue base resection using a coblator or the da Vinci robot) with or without nasal surgery (e.g., septoplasty, turbinoplasty) for OSA improvement (5); underwent preoperative nasopharyngoscopic examinations, such as MM and/or DISE; and (6) underwent postoperative PSG at 3 months or later. Patients with a history of previous airway surgery such as uvulopalatopharyngoplasty (UPPP), lateral pharyngoplasty (LP), or tonsillectomy prior to standard PSG were excluded.

Of the 137 patients who underwent sleep surgery during the period, 36 were excluded because they underwent palatal surgery (such as UPPP and LP) without the tongue base resection. Six patients were additionally excluded because of a history of previous airway surgery. Consequently, 95 OSA patients who underwent multilevel palate and tongue base surgery were included in this study.

All patients were divided into two groups according to their airway evaluation methods. Forty-seven patients comprised the MM group who underwent only MM for surgical indication, whereas 48 patients comprised the DISE group who underwent both MM and DISE.

\section{Airway evaluation}

All patients underwent upper airway evaluation using the Friedman staging system [11] and modified Mallampati grading [12]. Preoperative nasopharyngoscopic examination with MM and/or DISE in the supine position was performed for surgical decision [13, 14]. MM was performed basically for all patients prepared for sleep apnea surgery. However, if the results of the MM test were ambiguous for making surgical decisions or there was a mismatch between MM and PSG (e.g., in severe obstructive apnea without tongue base occlusion on $\mathrm{MM})$, the DISE test was further recommended and performed by a single surgeon (Dr. H.-J Cho). Patients aged $>60$ years or those who did not wish to undergo additional sedation testing did not undergo DISE, whereas some patients declined the test due to financial reasons.

DISE procedures were performed in the operating theater, whereas MM was performed at the outpatient clinic. Propofol alone, propofol-remifentanil combination, or dexmedetomidine-remifentanil combination was infused for inducing sleep apnea with proper monitoring of the participant according to the protocol, as reflected in our previous report [15].

The findings of MM and DISE were assessed using the modified VOTE classification system as suggested by Kezirian et al. [16]. The site and character of obstruction of the anatomical structure were assessed as follows: the velum, oropharyngeal lateral wall, tongue base, and epiglottis. The degree of occlusion, as revealed using nasopharyngoscopy, 
was categorized as 0 point $=$ no obstruction $(0-25 \%), 1$ point $=$ partial occlusion $(25-75 \%)$, and 2 points $=$ complete occlusion $(\geq 76 \%)$.

\section{Surgical decision protocol and surgical techniques}

Surgical decision for the tongue base surgery in the MM group was followed to the results of MM and that in the DISE group was followed to the results of DISE. Specific surgical decision protocol followed is as follows: nasal surgery, including septoplasty and turbinoplasty, was performed for patients with a deviated nasal septum and/or hypertrophied inferior turbinate found on nasal endoscopy and/or CT scan. Overlapping LP was performed for patients when the score of velum was $>1$ with a pattern of concentric or A-P/lateral wall obstruction on DISE and/or MM. Endoscopic coblator-assisted tongue base resection or transoral robotic tongue base resection was performed for patients who showed partial or complete obstruction at the tongue base level (MM or DISE score $\geq 1$ ). Surgical techniques of overlapping LP, endoscopic coblator-assisted tongue base resection, and transoral robotic tongue base resection were well described in our previous study [17]. Consequently, surgical procedure for tongue base resection was electively performed using a coblator or the da Vinci robot, with other palatal surgery (e.g., LP) and/or nasal surgery (e.g., septoplasty, turbinoplasty).

\section{Outcome measurement}

All patients underwent pre- and postoperative (at least 3 months later) standard PSG (Comet-PLUS ${ }^{\circ} \mathrm{XL}$, Grass Technologies, Warwick, RI, USA). Response rate was assessed based on three definitions: [1] $\mathrm{AHI}<5 / \mathrm{h}$ (complete resolution) [2]; AHI $<20 / \mathrm{h}$ with $\geq 50 \%$ AHI improvement (Sher criteria) [18]; and [3] $\geq 50 \%$ AHI improvement. The surgical success was defined according to the Sher criteria (definition 2). Additionally, AHI improvement (\%) was defined as (preoperative AHI - postoperative AHI) $\times 100 /$ preoperative AHI.

\section{Statistical analyses}

All continuous data are reported as mean \pm standard deviation. For comparison between the MM and DISE groups, $p$-values were calculated using independent or paired t-tests for continuous variables and using chisquare test for categorical variables. IBM SPSS version 23.0 statistical software (IBM Corp., Armonk, N.Y., USA) was used for statistical analysis of data. $p<0.05$ was considered statistically significant.

\section{Results}

Patient profiles

The demographic characteristics of participants are shown in Table 1. The mean age of patients in the MM group was $44.3 \pm 12.1$ years and that of patients in the DISE group was $41.8 \pm 12.2$ years $(p=0.308)$. Both groups were male-predominant (MM group, 85.1\%; DISE group, $87.5 \%, p=0.734$ ). The mean body mass index (BMI) of patients in the MM group $\left(25.3 \pm 3.2 \mathrm{~kg} / \mathrm{m}^{2}\right)$ was lower than that of patients in the DISE group $\left(26.3 \pm 3.2 \mathrm{~kg} / \mathrm{m}^{2}\right)$; however, the difference was not statistically significant $(p=0.130)$. There was also no statistically significant difference with respect to the tonsil grade $(p=0.076)$, Friedman staging $(p=0.056)$, and modified Mallampati grading $(p=0.570)$ between the two groups.

All participants in both groups underwent multilevel surgery of palatal surgery and tongue base surgery. For palatal surgery, LP was mainly performed in both groups (MM group $85.1 \%$, DISE group $100 \%, p=0.005$ ). Endoscopic coblator-assisted tongue base surgery was performed more than transoral robotic tongue base surgery in both groups (MM group 70.2\%, DISE group 81.3\%, $p=0.209$ ). About $60 \%$ of the participants in the MM group underwent nasal surgery (septoplasty $51.1 \%$, turbinoplasty $63.8 \%$ ), whereas less than half of those in the DISE group underwent nasal surgery (septoplasty 37.5\%, turbinoplasty $45.8 \%$ ). However, there was no significant difference in nasal surgery performed between both groups ( $p=0.078$, data not shown).

\section{Comparison of MM and DISE findings}

Results of MM and DISE of each patient in the DISE group were compared to identify their agreement for evaluation of the degree and pattern of obstruction at each anatomical level of the upper airway (Table 2). This comparison revealed fair agreement for the oropharyngeal lateral wall (kappa $=0.241, p=0.005)$, but did not show significant concordance at the velum, tongue base, and epiglottis. In 27 patients (56.3\%), the degree of

Table 1 Demographic characteristics of participants $(n=95)$

\begin{tabular}{llll}
\hline & MM group $(n=47)$ & DISE group $(n=48)$ & $P$-value \\
\hline Age, years & $44.3 \pm 12.1$ & $41.8 \pm 12.2$ & 0.308 \\
Sex, n (\%) & & & 0.734 \\
$\quad$ Male & $40(85.1 \%)$ & $42(87.5 \%)$ & \\
$\quad$ Female & $7(14.9 \%)$ & $6(12.5 \%)$ & \\
BMl, kg/m ${ }^{2}$ & $25.3 \pm 3.2$ & $26.3 \pm 3.2$ & 0.130 \\
NC & $38.7 \pm 8.6$ & $38.8 \pm 2.9$ & 0.957 \\
W/H ratio & $0.9 \pm 0.1$ & $0.9 \pm 0.1$ & 0.375 \\
Tonsil & $1.5 \pm 0.8$ & $1.8 \pm 0.9$ & 0.076 \\
MMP & $2.9 \pm 0.7$ & $3.0 \pm 0.8$ & 0.570 \\
Freidman stage & $2.6 \pm 0.6$ & $2.3 \pm 0.6$ & 0.056 \\
\hline
\end{tabular}

All data, except sex, are represented as mean \pm standard deviation Independent t-test was performed

Abbreviations: MM Muller's maneuver, DISE Drug-induced sleep endoscopy, BMI Body mass index, NC Neck circumference, W/H ratio Waist/hip ratio, MMP Modified Mallampati grade 
Table 2 Concordance between MM and DISE at the anatomic levels (DISE group, $n=48$ )

\begin{tabular}{llllll}
\hline $\begin{array}{l}\text { Obstruction } \\
\text { level }\end{array}$ & MM 0 & MM 1 & MM 2 & kappa & $\begin{array}{l}P \text { - } \\
\text { value }\end{array}$ \\
\hline VISE 0 & DISE 1 & DISE 2 & & \\
& $0(0 \%)$ & $20(41.7 \%)$ & $28(58.3 \%)$ & 0.115 & 0.087 \\
O & $0(0 \%)$ & $2(4.2 \%)$ & $46(95.8 \%)$ & & \\
& $3(62.5 \%)$ & $32(66.7 \%)$ & $12(25.0 \%)$ & 0.241 & $0.005^{* *}$ \\
$T$ & $7(14.6 \%)$ & $13(27.1 \%)$ & $28(58.3 \%)$ & & \\
& $11(22.9 \%)$ & $32(66.7 \%)$ & $5(10.4 \%)$ & 0.017 & 0.865 \\
E & $0(0 \%)$ & $24(50.0 \%)$ & $24(50.0 \%)$ & & \\
& $44(91.7 \%)$ & $2(4.2 \%)$ & $1(2.1 \%)$ & 0.122 & 0.202 \\
& $39(81.3 \%)$ & $3(6.3 \%)$ & $5(10.4 \%)$ & & \\
\hline
\end{tabular}

The degree of occlusion as per the nasopharyngoscopic study: $0=$ no obstruction (0-25\%), 1 = partial occlusion $(25-75 \%)$, and $2=$ complete occlusion ( $\geq 76 \%)$

Interpretation of kappa index: $<0$, no agreement; $0-0.19$, poor agreement; 0.20-0.39, fair agreement; $0.40-0.59$, moderate agreement; $0.60-0.79$, substantial agreement; $0.80-1.00$, almost perfect agreement Abbreviations: MM Muller's maneuver, DISE Drug-induced sleep endoscopy, $V$ Velum, $O$ Oropharynx lateral wall, $T$ Tongue base, $E$ Epiglottis ${ }^{*} p<0.05,{ }^{* *} p<0.01,{ }^{* * *} p<0.001$; statistical analyses were performed using Cohen's Kappa test

obstruction at the tongue base level assessed by DISE was greater than that assessed by MM, and in 19 patients $(39.6 \%)$, the results were the same. In only two patients (4.2\%), the degree of obstruction assessed by MM was higher than that assessed by DISE.

To compare the degree of occlusion between MM and DISE, we also compared the mean difference between the two methods in the DISE group using paired t-test (Table 3). All anatomical levels, except the epiglottis, showed significant mean differences between MM and DISE. The mean difference between MM and DISE was the greatest at the tongue base level (mean difference $0.63,95 \% \mathrm{CI}-0.83$ to $-0.42, p<0.001)$.

\section{Comparison of surgical outcomes between the MM and DISE groups}

Table 4 shows the results of pre- and postoperative PSG and Epworth sleepiness scale (ESS) scores in both groups. AHI, supine AHI, non-rapid eyeball movement AHI $\left(\mathrm{AHI} \mathrm{NREM}_{\mathrm{N}}\right)$, rapid eyeball movement $\mathrm{AHI}\left(\mathrm{AHI}_{\mathrm{REM}}\right)$, and nadir oxygen saturation (nadir $\mathrm{O}_{2}$ sat) were all significantly improved after the multilevel surgery in both groups. However, the ESS score was not significantly improved in the DISE group (preoperative vs. postoperative $=8.1 \pm 5.2$ vs. $7.4 \pm 5.0, p=0.165)$, whereas it significantly improved in the MM group $(9.4 \pm 4.9$ vs. $7.7 \pm 4.1, p=0.014)$.

The treatment response is presented in Fig. 1. Complete resolution (definition 1) was observed in $10.6 \%$ of patients in the MM group and in $16.7 \%$ in the DISE group ( $p=$ 0.393 ). The ratio of surgical success (definition 2) was $42.6 \%$ in the MM group and $45.8 \%$ in the DISE group $(p=0.748)$. The ratio of improved AHI $\geq 50 \%$ (definition 3) was $51.1 \%$ in the MM group and $47.9 \%$ in the DISE group $(p=0.759)$. However, these treatment responses were not significantly different between the groups. Improvement ratio of AHI was larger for patients in the DISE group $(48.3 \pm 35.4)$ than for those in the MM group (47.0 \pm 32.0$)$, but without statistical significance $(p=$ 0.852) (Fig. 2).

Results of identifying predictive factors that may affect surgical success are shown in Table 5 . There was no significant difference between the surgical success and surgical failure in age, BMI, neck circumference, $\mathrm{W} / \mathrm{H}$ ratio, modified Mallampati grade, Friedman stage, ESS, and several PSG parameters (such as AHI, supine AHI, $\mathrm{AHI}_{\mathrm{NREM}}, \mathrm{AHI}_{\mathrm{REM}}$, and nadir $\mathrm{O}_{2}$ sat). Tonsil grade was a significant predictive factor for surgical success in the analyses in both groups ( $p=0.004$ in the MM group, $p=$ 0.042 in the DISE group). Anatomical occlusion level was assessed using nasopharyngoscopic study for surgical decision (MM for the MM group, DISE for the DISE group). Interestingly, occlusion of the oropharyngeal lateral wall assessed by MM in the MM group showed a significant difference between surgical success and failure $(p=0.031)$, whereas that assessed by DISE in the DISE group did not show a significant difference $(p=$ 0.596). Other anatomical occlusion levels showed no significant differences between surgical success and failure (Fig. 3).

\section{Discussion}

In this study, our aim was to compare the outcomes of tongue base surgery based on MM and DISE in OSA

Table 3 Comparison of occlusion severity between MM and DISE according to the anatomic levels (DISE group, $n=48$ )

\begin{tabular}{lllll}
\hline $\begin{array}{l}\text { Obstruction } \\
\text { level }\end{array}$ & \multicolumn{3}{l}{ Nasopharyngoscopic study } & \multicolumn{2}{l}{$\begin{array}{l}\text { Mean difference } \\
(95 \% \mathrm{Cl})\end{array}$} & $P$-value \\
\cline { 2 - 3 } & $\mathrm{MM}$ & DISE & $-0.38(-0.52,-0.23)$ & $<0.001^{* * *}$ \\
\hline $\mathrm{V}$ & $1.58 \pm 0.50$ & $1.96 \pm 0.20$ & $-0.25(-0.44,-0.06)$ & $0.013^{*}$ \\
$\mathrm{~T}$ & $1.19 \pm 0.53$ & $1.44 \pm 0.74$ & $-0.63(-0.83,-0.42)$ & $<0.001^{* * *}$ \\
$\mathrm{E}$ & $0.92 \pm 0.54$ & $1.54 \pm 0.50$ & $-0.19(-0.41,-0.03)$ & 0.083 \\
\hline
\end{tabular}

Data are represented as mean \pm standard deviation

Abbreviations: MM Muller's maneuver, DISE Drug-induced sleep endoscopy,Cl Confidence interval), $V$ Velum, $O$ Oropharynx lateral wall, $T$ Tongue base, $E$ Epiglottis ${ }^{*} p<0.05,{ }^{* *} p<0.01,{ }^{* * *} p<0.001$; statistical analyses were performed using paired t-test 
Table 4 Evaluation of the pre- and postoperative polysomnographic findings and ESS scores in both groups

\begin{tabular}{|c|c|c|c|c|c|c|}
\hline & \multicolumn{3}{|c|}{ MM group $(n=47)$} & \multicolumn{3}{|c|}{ DISE group $(n=48)$} \\
\hline & Pre Op & Post Op & $P$-value & Pre Op & Post Op & $P$-value \\
\hline $\mathrm{AHI}$ & $39.7 \pm 18.8$ & $20.0 \pm 13.9$ & $<0.001^{* * *}$ & $48.1 \pm 20.7$ & $24.3 \pm 20.1$ & $<0.001^{* * *}$ \\
\hline Supine AHI & $53.5 \pm 22.5$ & $32.1 \pm 22.9$ & $0.002^{* *}$ & $62.0 \pm 21.5$ & $32.3 \pm 25.3$ & $<0.001^{* * *}$ \\
\hline$A H I_{\text {NREM }}$ & $39.3 \pm 19.2$ & $19.2 \pm 14.0$ & $<0.001^{* * *}$ & $49.8 \pm 22.2$ & $24.0 \pm 21.2$ & $<0.001^{* * *}$ \\
\hline$A H I_{\text {REM }}$ & $36.2 \pm 22.4$ & $18.7 \pm 13.3$ & $<0.001^{* * *}$ & $50.4 \pm 25.5$ & $24.5 \pm 20.2$ & $<0.001^{* * *}$ \\
\hline Nadir $\mathrm{O}_{2}$ saturation (\%) & $80.3 \pm 7.0$ & $83.3 \pm 8.3$ & $0.018^{*}$ & $77.7 \pm 9.1$ & $84.3 \pm 7.0$ & $<0.001^{* * *}$ \\
\hline ESS & $9.4 \pm 4.9$ & $7.7 \pm 4.1$ & $<0.014^{*}$ & $8.1 \pm 5.2$ & $7.4 \pm 5.0$ & 0.165 \\
\hline
\end{tabular}

Data are represented as mean \pm standard deviation

Abbreviations: MM Muller's maneuver, DISE Drug-induced sleep endoscopy, AHI Apnea-hypopnea index, NREM Non-rapid eye movement sleep, REM Rapid eye movement sleep, ESS Epworth sleepiness scale

${ }^{*} p<0.05,{ }^{* *} p<0.01,{ }^{* * *} p<0.001$; statistical analyses were performed using paired t-test

patients. Furthermore, we tried to identify factors predictive of surgical success in nasopharyngoscopic study.

Identification of the airway obstruction site is important to determine the method of surgical treatment in OSA patients. OSA patients can exhibit multilevel obstruction, including those at the nasal and retrolingual levels. In 1984, Fusita [19] firstly described different anatomic levels of obstruction in OSA patients. He pointed out that most nonresponders to UPPP have multilevel obstruction, such as combined oropharyngeal and hypopharyngeal segments. Outcomes of sleep surgery are affected by the obstruction site and surgical techniques [18, 20,21].

Surgical planning mainly depends on the identification of the site and degree of obstruction. Endoscopic examination has been considered as an indispensable technique to identify the exact site of obstruction in OSA patients. Awake fiber-optic nasal endoscopic evaluation with $M M$ has been performed commonly at outpatient clinics. However, it is considered less effective in predicting surgical outcomes because it tends to underestimate the collapse at the retrolingual level and cannot assess the actual state of sleep [22, 23]. To overcome these shortcomings, DISE has been widely used over the last 20 years. Several recent studies have tried to identify concordance between the MM and DISE findings. These studies have reported significant concordance between MM and DISE for evaluating obstruction at the retropalatal level and lateral wall of the oropharynx, but the results for obstruction at the tongue base level were discordant between MM and DISE [7, 8]. Moreover, these studies also reported that MM has an increased tendency of underestimating obstruction compared with DISE. Our data also showed weak concordance at the tongue base level, and the tendency of MM to underestimate (Tables 2 and 3). From these results, one might expect that DISE would be more sensitive than MM, especially for detecting occlusion at the tongue base level.

Several studies have shown the impact of DISE in planning the surgical method and its outcome in OSA patients. As we have mentioned above, some recent studies have reported that surgical plan based on awake examination was changed after performing DISE in 40$50 \%$ of patients $[9,10]$. There are several conflicting reports about the surgical outcomes of DISE. Some studies have reported that DISE-based diagnosis increases the success rates of sleep surgery [24, 25]. In a multicenter study on OSA patients with single- or multi-level occlusion, the surgical outcome of DISE was significantly worse than that of MM [26]. However, most of these previous studies compared the surgical outcomes based on MM and DISE, with respect to UPPP (including

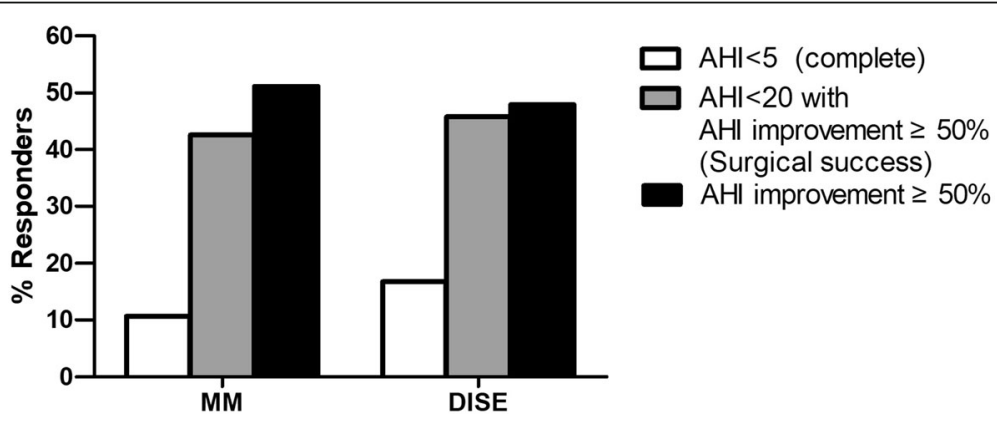

Fig. 1 Comparison of response rates after tongue base surgery according to MM and DISE. Response rate was assessed based on three definitions: [1] AHI <5/h [2]; $\mathrm{AHI}<10 / \mathrm{h}$ with $\geq 50 \% \mathrm{AHI}$ improvement [3]; $\geq 50 \% \mathrm{AHI}$ improvement. There was no significant difference in the response rates between MM and DISE. Abbreviations: MM, Muller's maneuver; DISE, drug-induced sleep endoscopy; AHI, apnea-hypopnea index. 


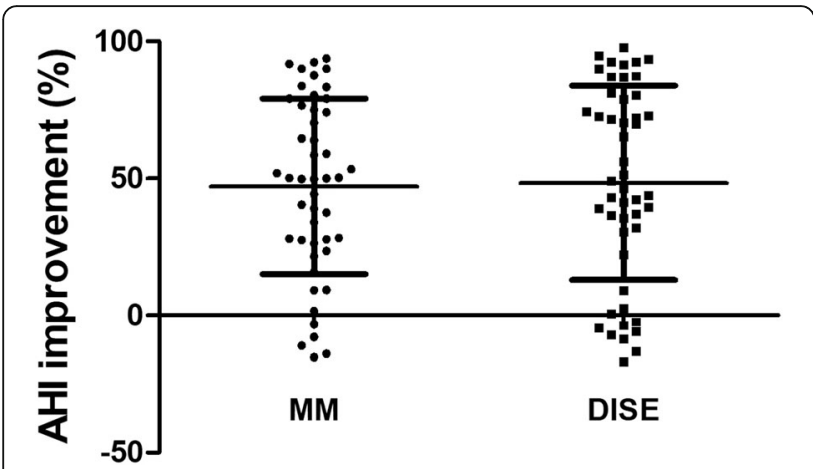

Fig. 2 Comparison of AHI improvement after OSA surgery according to $\mathrm{MM}$ and DISE. There was no significant difference in AHI improvement between MM and DISE ( $p=0.852$ ). Abbreviations: AHI, apnea-hypopnea index; OSA, obstructive sleep apnea; MM, Muller's maneuver; DISE, drug-induced sleep endoscopy.

tonsillectomy), and did not focus on the tongue base surgery [13]. In this study, we tried to obtain results in a more controlled population by including only patients with multilevel occlusion.

As per our findings, the success rate of tongue base surgery based on DISE was slightly higher than that of surgery based on MM; however, the difference was not statistically significant. Results of the AHI reduction ratio in patients after the tongue base surgery based on both techniques were also not significantly different. There are several possible reasons why the surgical outcomes were not statistically different between the MM and DISE groups. First, DISE was basically recommended for patients with a mismatch on MM and PSG results in our study, indicating that patients in the DISE group might have more complex OSA than those in the MM group. Although there was no significant difference in demographic data between the MM and DISE groups (Table 1), there is a potential for selection bias that can lead to these negative consequences. Second, DISE does not reflect the actual sleep state of the airway as it changes the sleep architecture. For example, only nonREM sleep status is generally attained by DISE [27]. Moreover, the duration of examination is not identical to that of the natural physiological sleep. Third, tongue base resection surgery might not effectively improve the airway obstruction at the tongue base level. It has been expected that DISE can discriminate occlusion of the tongue base more sensitively and with a higher quality

Table 5 Predictive values for surgical success

\begin{tabular}{|c|c|c|c|c|c|c|c|}
\hline & & \multicolumn{3}{|l|}{ MM group } & \multicolumn{3}{|l|}{ DISE group } \\
\hline & & $\begin{array}{l}\text { Surgical success }(n= \\
20 \text { ) }\end{array}$ & $\begin{array}{l}\text { Surgical failure }(n= \\
27)\end{array}$ & $\begin{array}{l}P \text { - } \\
\text { value }\end{array}$ & $\begin{array}{l}\text { Surgical success ( } n= \\
22 \text { ) }\end{array}$ & $\begin{array}{l}\text { Surgical failure }(n= \\
\text { 26) }\end{array}$ & $\begin{array}{l}P \\
\text {-value }\end{array}$ \\
\hline Sex, male (\%) & & 15 (75\%) & $25(92.6 \%)$ & 0.094 & $20(90.1 \%)$ & $22(84.6 \%)$ & 0.511 \\
\hline Age & & $43.7 \pm 11.2$ & $44.8 \pm 13.0$ & 0.768 & $38.5 \pm 10.4$ & $44.5 \pm 13.2$ & 0.090 \\
\hline BMl & & $25.4 \pm 2.8$ & $25.5 \pm 3.4$ & 0.852 & $26.3 \pm 3.5$ & $26.3 \pm 3.0$ & 0.958 \\
\hline $\mathrm{NC}$ & & $40.1 \pm 12.9$ & $37.7 \pm 2.5$ & 0.371 & $39.0 \pm 2.7$ & $38.7 \pm 3.2$ & 0.703 \\
\hline $\mathrm{W} / \mathrm{H}$ ratio & & $0.91 \pm 0.06$ & $0.92 \pm 0.07$ & 0.649 & $0.88 \pm 0.07$ & $0.92 \pm 0.05$ & $0.018^{*}$ \\
\hline Tonsil grade & & $1.9 \pm 0.8$ & $1.2 \pm 0.7$ & $0.004^{* *}$ & $2.1 \pm 0.9$ & $1.6 \pm 0.8$ & $0.042^{*}$ \\
\hline MMP & & $3.0 \pm 0.8$ & $2.9 \pm 0.7$ & 0.670 & $3.1 \pm 0.6$ & $2.9 \pm 0.9$ & 0.583 \\
\hline Friedman staging & & $2.5 \pm 0.8$ & $2.8 \pm 0.4$ & 0.343 & $2.2 \pm 0.6$ & $2.4 \pm 0.8$ & 0.486 \\
\hline $\mathrm{AHI}$ & & $37.9 \pm 2.4$ & $41.0 \pm 17.8$ & 0.581 & $53.2 \pm 21.7$ & $43.7 \pm 19.2$ & 0.115 \\
\hline Supine AHI & & $53.6 \pm 24.0$ & $53.7 \pm 21.4$ & 0.996 & $66.0 \pm 19.2$ & $58.7 \pm 23.0$ & 0.259 \\
\hline$A H I_{\text {NREM }}$ & & $37.0 \pm 19.0$ & $41.9 \pm 19.2$ & 0.405 & $55.4 \pm 22.7$ & $45.0 \pm 21.1$ & 0.115 \\
\hline $\mathrm{AH} \mathrm{REM}_{\mathrm{RE}}$ & & $37.5 \pm 29.2$ & $33.4 \pm 21.4$ & 0.603 & $53.7 \pm 25.3$ & $46.8 \pm 24.8$ & 0.378 \\
\hline Nadir $\mathrm{O}_{2}$ sat, (\%) & & $81.4 \pm 4.8$ & $79.4 \pm 8.2$ & 0.294 & $77.1 \pm 8.7$ & $78.3 \pm 9.5$ & 0.632 \\
\hline ESS & & $9.7 \pm 5.1$ & $9.4 \pm 4.8$ & 0.850 & $8.5 \pm 5.2$ & $7.8 \pm 5.2$ & 0.682 \\
\hline \multirow{4}{*}{$\begin{array}{l}\text { Nasopharyngoscopic } \\
\text { evaluation }^{\text {a }}\end{array}$} & V & $1.8 \pm 0.6$ & $1.5 \pm 0.5$ & 0.143 & $2.0 \pm 0.0$ & $1.9 \pm 0.3$ & 0.161 \\
\hline & $\mathrm{O}$ & $1.2 \pm 0.6$ & $0.9 \pm 0.5$ & $0.031^{*}$ & $1.5 \pm 0.7$ & $1.4 \pm 0.8$ & 0.596 \\
\hline & $\mathrm{T}$ & $1.1 \pm 0.5$ & $0.8 \pm 0.6$ & 0.112 & $1.5 \pm 0.5$ & $1.6 \pm 0.5$ & 0.275 \\
\hline & $E$ & $0.1 \pm 0.3$ & $0.04 \pm 0.2$ & 0.394 & $0.4 \pm 0.7$ & $0.2 \pm 0.5$ & 0.186 \\
\hline Nasal surgery, n (\%) & & $11(55.0 \%)$ & $19(70.3 \%)$ & 0.278 & $11(50 \%)$ & 11 (42.3\%) & 0.594 \\
\hline
\end{tabular}

Abbreviations: MM Muller's maneuver, DISE Drug-induced sleep endoscopy, BMI Body mass index, NC Neck circumference, W/H ratio Waist/hip ratio, MMP Modified Mallampati grade, AHI Apnea-hypopnea index, NREM Non-rapid eye movement sleep, REM Rapid eye movement sleep, Nadir $\mathrm{O}_{2}$ sat Nadir $\mathrm{O}_{2}$ saturation, ESS Epworth sleepiness scale, $V$ Velum, $O$ Oropharynx lateral wall, $T$ Tongue base, $E$ Epiglottis

${ }^{*} p<0.05,{ }^{* *} p<0.01$; statistical analyses were performed by independent t-test

a Scoring occlusion level was represented according to the surgical decision methods for each group; by MM for MM group and by DISE for DISE group 


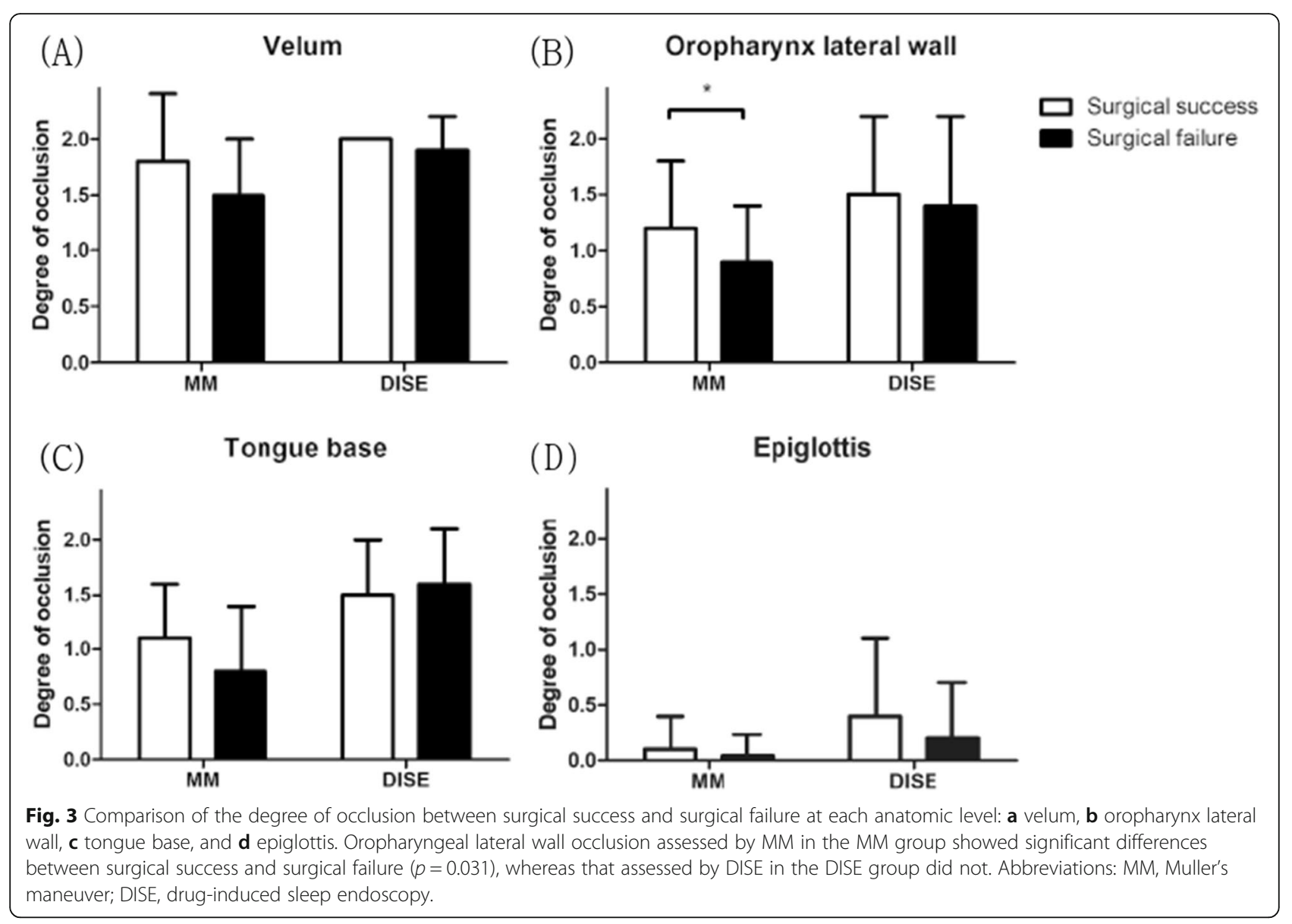

than MM, which can lead to a better surgical outcome. However, according to our results, there were no significant differences in the surgical outcomes between the two methods. Because the indication of tongue base surgery has not been strictly established, it may be necessary to clarify the criteria for patient selection to improve the surgical outcomes. Finally, there is also a possibility that the evaluative method or grading system used in the DISE and MM tests is not elaborate and may not accurately reflect the collapsible pattern or location. Judging from our results and other those of previous studies, there is still not enough evidence to conclude that surgical results of DISE are superior to those of the awake test.

In the present study, the tonsil size was statistically related to surgical success. Interestingly, the mean value of MM findings on the oropharyngeal lateral wall occlusion was significantly different between surgical success and surgical failure, whereas the mean value of DISE findings was not. The degree of occlusion of the oropharyngeal lateral wall can be generally considered to be related to the tonsil size. Therefore, these findings of MM on the oropharyngeal lateral wall seem reasonable. This mismatch between the MM and DISE findings suggests that the findings of DISE might be more exaggerated in measuring occlusion of the oropharyngeal lateral wall than those of MM.

There were also some limitations to our study. This study was retrospective in nature, so it might not have been as well controlled as a prospective study. Moreover, there was no statistical difference in surgical outcomes between the MM and DISE groups. This may be attributed to the small number of patients that were enrolled in this study. Therefore, prospective studies are needed in the future to more accurately evaluate the therapeutic value of DISE in tongue base surgery.

\section{Conclusions}

This study was conducted to compare the surgical outcomes of MM and DISE, which are widely used to determine sleep surgery. From this study on the tongue base surgery, MM and DISE findings showed low agreement. DISE might affect the surgical decision in OSA patients compared to MM. However, there was no significant difference in the surgical results between MM and DISE. However, because this study is of the limited outcome under a retrospective design, there is not enough evidence to conclude on the comparison of surgical 
outcomes between MM and DISE. We look forward to a follow-up study comparing the surgical outcomes of $\mathrm{MM}$ and DISE under a prospective design.

\section{Abbreviations}

AHI: Apnea-hypopnea index; BMI: Body mass index; CPAP: Continuous positive airway pressure; DISE: Drug-induced sleep endoscopy; E: Epiglottis; ESS: Epworth sleepiness scale; LP: Lateral pharyngoplasty; MM: Muller's maneuver; O: Oropharynx lateral wall; OSA: Obstructive sleep apnea; PSG: Polysomnography; REM: Rapid eyeball movement; T: Tongue base; UPPP: Uvulopalatopharyngoplasty; V: Velum

\section{Acknowledgments}

Not applicable.

\section{Author's contributions}

Drs J.-G Ha and H.-J Cho had full access to all of the data in the study and take responsibility for the integrity of the data and the accuracy of the data analysis. Study concept and design: J.-G Ha, H.-J Cho. Acquisition, analysis, or interpretation of data: Y Lee, JS Nam, JJ Park. Drafting of the manuscript: J.-G $\mathrm{Ha}$. Critical revision of the manuscript for important intellectual content: C.-H Kim, J.-H Yoon, H.-J Cho. Statistical analysis: J.-G Ha, JS Nam. Administrative, technical, or material support: J.-G Ha, Y Lee, JS Nam, JJ Park, H.-J Cho. Study supervision: H.-J Cho. The author(s) read and approved the final manuscript.

\section{Funding}

This research was supported by the Basic Science Research Program through the National Research Foundation of Korea (NRF) funded by the Ministry of Education (NRF-2018R1D1A1A02049236) to H.J. Cho and by the Bio \& Medical Technology Development Program of the NRF funded by the Ministry of Science, ICT \& Future Planning (NRF-2016M3A9D5A01952414) and by the NRF grant funded by the Korean Government (MSIP) (No. 2016R1A5A2008630). This study was also supported by a Faculty Research Grant of Yonsei University College of Medicine for (6-2018-0167).

\section{Availability of data and materials}

The datasets analyzed during the current study are available from the corresponding author on reasonable request.

\section{Ethics approval and consent to participate}

This retrospective study was reviewed and approved by the Institutional Review Board of Severance Hospital, South Korea (4-2018-0861). Informed consent was waived by the institutional review board because of the retrospective study design. This study was also performed according to the Declaration of Helsinki.

\section{Consent for publication}

Not applicable.

\section{Competing interests}

The authors declare that they have no competing interests.

\section{Author details}

'Department of Otorhinolaryngology, Yonsei University College of Medicine, 50-1 Yonsei-ro, Seodaemun-gu, Seoul 03722, South Korea. ${ }^{2}$ The Airway Mucus Institute, Yonsei University College of Medicine, Seoul, South Korea. ${ }^{3}$ Korea Mouse Sensory Phenotyping Center, Yonsei University College of Medicine, Seoul, South Korea. ${ }^{4}$ Medical Research Center, Yonsei University College of Medicine, Seoul, South Korea.

\section{Received: 23 May 2019 Accepted: 17 February 2020}

Published online: 24 February 2020

\section{References}

1. Ruehland WR, Rochford PD, O'Donoghue FJ, Pierce RJ, Singh P, Thornton AT. The new AASM criteria for scoring hypopneas: impact on the apnea hypopnea index. Sleep. 2009;32(2):150-7.

2. Epstein LJ, Kristo D, Strollo PJ Jr, Friedman N, Malhotra A, Patil SP, et al. Clinical guideline for the evaluation, management and long-term care of obstructive sleep apnea in adults. J Clin Sleep Med. 2009;5(3):263-76.
3. Marin JM, Carrizo SJ, Vicente E, Agusti AG. Long-term cardiovascular outcomes in men with obstructive sleep apnoea-hypopnoea with or without treatment with continuous positive airway pressure: an observational study. Lancet. 2005;365(9464):1046-53.

4. Shelgikar AV, Aronovich S, Stanley JJ. Multidisciplinary alternatives to CPAP program for CPAP-intolerant patients. J Clin Sleep Med. 2017;13(3):505-10.

5. Terris DJ, Hanasono MM, Liu YC. Reliability of the Muller maneuver and its association with sleep-disordered breathing. Laryngoscope. 2000;110(11): 1819-23.

6. De Vito A, Carrasco Llatas M, Vanni A, Bosi M, Braghiroli A, Campanini A, et al. European position paper on drug-induced sedation endoscopy (DISE). Sleep Breath. 2014;18(3):453-65.

7. Cavaliere M, Russo F, lemma M. Awake versus drug-induced sleep endoscopy: evaluation of airway obstruction in obstructive sleep apnea/ hypopnoea syndrome. Laryngoscope. 2013;123(9):2315-8.

8. Jung AR, Koh TK, Kim SJ, Lee KH, Cho JS, Kim SW. Comparison of level and degree of upper airway obstruction by Muller's maneuver and druginduced sleep endoscopy in obstructive sleep apnea patients. Auris Nasus Larynx. 2017:44(5):571-5.

9. Fernandez-Julian E, Garcia-Perez MA, Garcia-Callejo J, Ferrer F, Marti F, Marco J. Surgical planning after sleep versus awake techniques in patients with obstructive sleep apnea. Laryngoscope. 2014;124(8):1970-4.

10. Certal VF, Pratas R, Guimaraes L, Lugo R, Tsou Y, Camacho M, et al. Awake examination versus DISE for surgical decision making in patients with OSA: a systematic review. Laryngoscope. 2016;126(3):768-74.

11. Friedman M, Tanyeri H, La Rosa M, Landsberg R, Vaidyanathan K, Pieri S, et al. Clinical predictors of obstructive sleep apnea. Laryngoscope. 1999; 109(12):1901-7.

12. Samsoon GL, Young JR. Difficult tracheal intubation: a retrospective study. Anaesthesia. 1987;42(5):487-90

13. Blumen M, Bequignon E, Chabolle F. Drug-induced sleep endoscopy: a new gold standard for evaluating OSAS? Part II: results. Eur Ann Otorhinolaryngol Head Neck Dis. 2017;134(2):109-15.

14. Sher AE, Thorpy MJ, Shprintzen RJ, Spielman AJ, Burack B, McGregor PA. Predictive value of Muller maneuver in selection of patients for uvulopalatopharyngoplasty. Laryngoscope. 1985;95(12):1483-7.

15. Cho JS, Soh S, Kim EJ, Cho HJ, Shin S, Kim HJ, et al. Comparison of three sedation regimens for drug-induced sleep endoscopy. Sleep Breath. 2015; 19(2):711-7.

16. Kezirian EJ, Hohenhorst W, de Vries N. Drug-induced sleep endoscopy: the VOTE classification. Eur Arch Otorhino-Laryngology. 2011;268(8):1233-6.

17. Park DY, Chung HJ, Park SC, Kim JW, Yoon JH, Lee JG, et al. Surgical outcomes of overlapping lateral pharyngoplasty with or without coblator tongue base resection for obstructive sleep apnea. Eur Arch Otorhinolaryngol. 2018;275(5):1189-96.

18. Sher AE, Schechtman KB, Piccirillo JF. The efficacy of surgical modifications of the upper airway in adults with obstructive sleep apnea syndrome. Sleep. 1996;19(2):156-77.

19. Fujita S. UPPP for sleep apnea and snoring. Ear, Nose Throat J. 1984;63(5): $227-35$.

20. Iwanaga K, Hasegawa K, Shibata N, Kawakatsu K, Akita Y, Suzuki K, et al. Endoscopic examination of obstructive sleep apnea syndrome patients during drug-induced sleep. Acta Otolaryngol Suppl. 2003;550:36-40.

21. Aktas O, Erdur O, Cirik AA, Kayhan FT. The role of drug-induced sleep endoscopy in surgical planning for obstructive sleep apnea syndrome. Eur Arch Otorhinolaryngol. 2015;272(8):2039-43.

22. Doghramji $\mathrm{K}$, Jabourian ZH, Pilla M, Farole A, Lindholm RN. Predictors of outcome for uvulopalatopharyngoplasty. Laryngoscope. 1995;105(3 Pt 1): $311-4$.

23. Petri N, Suadicani P, Wildschiodtz G, Bjorn-Jorgensen J. Predictive value of Muller maneuver, cephalometry and clinical features for the outcome of uvulopalatopharyngoplasty. Evaluation of predictive factors using discriminant analysis in 30 sleep apnea patients. Acta Otolaryngol. 1994;114(5):565-71.

24. Hessel NS, de Vries N. Results of uvulopalatopharyngoplasty after diagnostic workup with polysomnography and sleep endoscopy: a report of 136 snoring patients. Eur Arch Otorhinolaryngol. 2003;260(2):91-5.

25. Huntley C, Chou D, Doghramji K, Boon M. Preoperative drug induced sleep endoscopy improves the surgical approach to treatment of obstructive sleep apnea. Ann Otol Rhinol Laryngol. 2017;126(6):478-82.

26. Pang KP, Baptista PM, Olszewska E, Braverman I, Carrasco-Llatas M, Kishore S, et al. Does drug-induced sleep endoscopy affect surgical outcome? A 
multicenter study of 326 obstructive sleep apnea patients. Laryngoscope. 2020;130(2):551-5.

27. Rabelo FA, Kupper DS, Sander HH, Fernandes RM, Valera FC.

Polysomnographic evaluation of propofol-induced sleep in patients with

respiratory sleep disorders and controls. Laryngoscope. 2013;123(9):2300-5.

\section{Publisher's Note}

Springer Nature remains neutral with regard to jurisdictional claims in published maps and institutional affiliations.

Ready to submit your research? Choose BMC and benefit from:

- fast, convenient online submission

- thorough peer review by experienced researchers in your field

- rapid publication on acceptance

- support for research data, including large and complex data types

- gold Open Access which fosters wider collaboration and increased citations

- maximum visibility for your research: over $100 \mathrm{M}$ website views per year

At $\mathrm{BMC}$, research is always in progress.

Learn more biomedcentral.com/submissions 